\title{
Changes in Lectin Binding Patterns of Mouse Male Germ Cells (Gonocytes) during Prespermatogenesis
}

\author{
Reiko NAGANO, Xiuhua SUN, Masamichi KUROHMARU and Yoshihiro HAYASHI \\ Department of Veterinary Anatomy, Graduate School of Agricultural and Life Sciences, The University of Tokyo, Bunkyo-ku, Tokyo 113- \\ 8657, Japan
}

(Received 26 October 1998/Accepted 19 December 1998) ABSTRACT. The distribution of sugar residues in gonocytes of the differentiating mouse testis was examined by light microscopy using 22
different kinds of lectins. Characteristic binding patterns of sWGA, VVA, and LEA in gonocytes were observed during prespermatogenesis.
sWGA preferentially bound to the cytoplasm and plasma membrane of gonocytes on 16.5 days post coitus (dpc). Its reaction decreased
thereafter and almost disappeared on 1.5 days post partum $(\mathrm{dpp})$, but reaction reappeared on $4.5 \mathrm{dpp}$ and continued until $6.5 \mathrm{dpp}$. The
VVA reaction was recognized in a few gonocytes on $0.5 \mathrm{dpp}$, and remained strong until $6.5 \mathrm{dpp}$. LEA reacted strongly in the plasma
membrane and cytoplasm of gonocytes from $0.5 \mathrm{dpp}$ to $6.5 \mathrm{dpp}$. The present study indicates that sWGA, VVA, and LEA are useful
markers for gonocytes, and the appearance or disappearance of sWGA and VVA may be related to the differentiation of gonocytes during
prespermatogenesis.- KEY wORDs: differentiation, glycoconjugate, gonocyte, lectin, prespermatogenesis

In an early postnatal life, the cells of the seminiferous epithelium undergo developmental changes that are ultimately critical for establishment of spermatogenesis. In particular, the gonocytes (precursors of germ cell lineage) in the seminiferous tubule resume their mitotic activity, relocate on the basement membrane, and give rise to spermatogonia. At first, they proliferate until about 14.5 days post coitus (dpc) to $16.5 \mathrm{dpc}[18,27,29]$, and then are arrested in the G1 phase of the cell cycle. After birth, the gonocytes reinitiate to divide [13, 21]. They extend cytoplasmic processes to the basement membrane and move from the central region toward the base [8, 25]. Thus, spermatogenic cells are placed in an environment likely to be crucial for their further maturation [7, 17, 20, 24]. These stages are called prespermatogenesis [10, 30]. Recent investigations indicate that the change in carbohydrate distribution in the cytoplasm and plasma membrane of various cells is strongly related to cellular differentiation and to cell-to-cell interactions [9, 23]. Lectins, which bind to specific sugar residues, have been used as potent probes for identification of the carbohydrate molecules on cell surface and in cytoplasmic organelles. Important roles of the complex carbohydrates on the cell surface have been shown by lectin histochemistry [5, 12, 22, 26]. In developing gonads, stage-specific changes in carbohydrates on the surface of germ cells have been reported using lectins [2-4, 14] or monoclonal antibodies such as SSEA-1, EMA1, EMA-6, Le ${ }^{\mathrm{x}}$, EE2 and 4C9 $[6,11,16,19,31]$ as probes. So far, however, little information has been available on the lectin binding pattern during prespermatogenesis. In the present study, the changes in the distribution of surface and cytoplasmic glycoconjugates in mouse gonocytes were examined by light microscopy using lectins as probes.

\section{MATERIALS AND METHODS}

Preparation of animals: Virgin female 8- to 12-week-old mice from the inbred strain C57BL/6J were mated with males of the same strain. All animals were maintained in our laboratory. They were housed on a standard program of $14 \mathrm{hr}$ light: $10 \mathrm{hr}$ dark and were given food and water $\mathrm{ad}$ libitum. Females were placed with males overnight and the presence or absence of spermatozoa in the vaginal smear was examined in the next morning. The day when spermatozoa were found was designated as $0.5 \mathrm{dpc}$ of gestation. Testes were obtained from fetuses $16.5 \mathrm{dpc}$ to $18.5 \mathrm{dpc}$ and new borns 0.5 day post partum (dpp) to 6.5 dpp.

Staining with lectins for light microscopy: The lectin probes used in this study were Concanavalin A agglutinin (ConA, Canavalia ensiformis), Bauhinia purpurea agglutinin (BPA), succinylated wheat germ agglutinin (sWGA), wheat germ agglutinin (WGA, Triticum vulgaris), Griffonia simplicifolia agglutinin II (GS-II), Datura stramonium agglutinin (DSA), peanut agglutinin (PNA, Arachis hypogea), Griffonia simplicifolia agglutinin I (GSI), soybean agglutinin (SBA), Dolichos biflorus agglutinin (DBA), Ulex europeus agglutinin (UEA-I), Bandeiraea simplicifolia agglutinin I (BSA-I), Pisum sativum agglutinin (PSA), Lens culinaris agglutinin (LCA), Phaseolus vulgaris Erythro agglutinin (PHA-E), Phaseolus vulgaris Leuco agglutinin (PHA-L), Sophora japonica agglutinin (SJA), Bandeiraea simplicifolia agglutinin II (BSA-II), Erythrina cristagalli agglutinin (ECA), Lycopersicon esculentum (tomato) agglutinin (LEA), Vicia villosa agglutinin (VVA), and Jacalin. Biotinyl lectins (Vector Laboratories, Burlingame, CA, U.S.A.) were used along with avidinbiotinyl peroxidase complexes (ABC, Vector Lab.). Testes 
were fixed in $10 \%$ formalin containing $2 \%$ calcium acetate for 2 days at $4^{\circ} \mathrm{C}$. They were then dehydrated in a graded series of ethanol, infiltrated in xylene and embedded in paraffin. Deparaffinized sections ( $4 \mu \mathrm{m}$ thick) were rehydrated and treated with $1 \%$ bovine serum albumin (BSA) in $10 \mathrm{mM}$ phosphate-buffered saline (PBS), pH 7.2. The sections were incubated with biotinyl lectins $(25 \mu \mathrm{g} /$ $\mathrm{m} l$ ) in $0.1 \%$ BSA-PBS for $60 \mathrm{~min}$, and then washed with PBS. They were incubated with $\mathrm{ABC}$ for $30 \mathrm{~min}$. After a rinse with PBS again, they were immersed in 3,3'diaminobenzidine (DAB, $0.2 \mathrm{mg} / \mathrm{m} l)-\mathrm{H}_{2} \mathrm{O}_{2}(0.005 \%)$ for 10 min, rinsed with distilled water, dehydrated, mounted and observed by light microscopy.

Control experiments: As control experiments to clarify binding specificity, deparaffinized sections were preincubated with appropriate hapten sugars for each lectin and then incubated in lectin solution containing hapten sugars. Non-specific staining was also checked by incubation with $\mathrm{ABC}$ and $\mathrm{DAB}-\mathrm{H}_{2} \mathrm{O}_{2}$ solution.

\section{RESULTS}

As shown in Table 1, specific binding patterns were observed in three lectins (sWGA, VVA, and LEA). The detailed binding pattern of each lectin is described below. On $16.5 \mathrm{dpc}$, sWGA showed a granule-like reaction in the cytoplasm of gonocytes. The plasma membrane of gonocytes was also positive (Fig. 1A). The sWGA reaction decreased on $0.5 \mathrm{dpp}$ (Fig. 1B), and almost disappeared on $1.5 \mathrm{dpp}$. However, the reaction reappeared in the cytoplasm and plasma membrane of gonocytes on $4.5 \mathrm{dpp}$ and continued until $6.5 \mathrm{dpp}$ (Fig. 1C). VVA reacted in only a part of interstitium on $16.5 \mathrm{dpc}$ (Fig. 2A). The VVA reaction was observed in the cytoplasm (granule-like) and plasma membrane of gonocytes on $0.5 \mathrm{dpp}$ (Fig. 2B) strongly positive in the cytoplasm of gonocytes on $4.5 \mathrm{dpp}$ (Fig. 2C). The reaction remained to be positive until 6.5 dpp. LEA reacted strongly with the plasma membrane and cytoplasm of gonocytes on $16.5 \mathrm{dpc}$ (Fig. 3A, B). The LEA-positive reaction continued until $6.5 \mathrm{dpp}$ (Fig. 3C). A weak reaction of sWGA and LEA was occasionally observed in the plasma membrane of Sertoli cells during prespermatogenesis. BSA-I, PHA-L, PHA-E, DSA, ConA, WGA, ECA, PSA, and LCA showed a positive reaction in the plasma membrane and cytoplasm of gonocytes, Sertoli cells and other somatic cells. DBA, BPA, SBA, GS-I, GSII, Jacalin, PNA, SJA, UEA-I, and BSA-II showed no definite reaction throughout prespermatogenesis. sWGA, VVA, and LEA- binding were completely inhibited by incubation in the presence of GalNac and GlcNac (Figs. $1 \mathrm{D}, 2 \mathrm{D}$, and 3D) or by treatment with $\alpha$-amylase $(1 \mathrm{mg} / \mathrm{ml}$, $37^{\circ} \mathrm{C}, 1 \mathrm{hr}$;Wako, Osaka, Japan).

\section{DISCUSSION}

In the present study, changes in lectin binding patterns in mouse gonocytes during prespermatogenesis were examined.
Table 1. Changes in reactivity of lectin bindings to gonocytes during prespermatogenesis

\begin{tabular}{|c|c|c|c|c|c|c|c|c|c|c|}
\hline & \multicolumn{3}{|c|}{ Fetal stage (dpc) } & \multicolumn{7}{|c|}{ Postnatal stage (dpp) } \\
\hline & 16.5 & 17.5 & 18.5 & 0.5 & 1.5 & 2.5 & 3.5 & 4.5 & 5.5 & 6.5 \\
\hline s & ++ & ++ & + & $-\sim \pm$ & - & - & - & 1 & + & + \\
\hline VVA & - & - & - & $-\sim \pm$ & + & + & + & + & + & + \\
\hline LEA & ++ & ++ & ++ & ++ & ++ & ++ & ++ & ++ & ++ & ++ \\
\hline
\end{tabular}

- (negative) $\sim+$ (positive)

Some lectins bound to gonocytes (Table 1). The binding of the lectins examined in this study was classified into five different patterns. (1) sWGA reaction in gonocytes appeared from $16.5 \mathrm{dpc}$ to $0.5 \mathrm{dpp}$, once disappeared on $1.5 \mathrm{dpp}$, and then reappeared on $4.5 \mathrm{dpp}$ and continued until $6.5 \mathrm{dpp}$. (2) VVA reaction appeared in gonocytes on $0.5 \mathrm{dpp}$ and continued until $6.5 \mathrm{dpp}$. (3) LEA reacted strongly in gonocytes throughout the stages examined. (4) BSA-I, PHA-L, PHA-E, DSA, ConA, WGA, ECA, PSA, and LCA reacted in most of the gonocytes and Sertoli cells. (5) DBA, BPA, SBA, GS-I, GS-II, Jacalin, PNA, SJA, and BSA-II showed no definite reaction throughout the stages examined. Because sWGA and LEA have specific affinity to $\beta(1,4)$ GlcNAc, and VVA has specific affinity to GalNAc $\underline{\alpha}_{3} \mathrm{Gal}$, certain glycoconjugates containing $\beta(1,4)$ GlcNAc and GalNAc $\underline{\alpha}_{3} \mathrm{Gal}$ residues are expressed in the cytoplasm and plasma membrane of gonocytes during prespermatogenesis. The changes in sWGA-and VVA-binding may especially relate to differentiation of gonocytes because the gonocytes begin to reproliferate from $1.5 \mathrm{dpp}$ and differentiate into type A spermatogonia on $4.5 \mathrm{dpp}$ [27]. In addition, these three lectins should be a selective marker for gonocytes. According to reports using the monoclonal antiserum against Forssman antigenic determinant (FA), monoclonal antigen 4B6, 3E10, these antigens appeared in most of mouse germ cells in fetal stages and disappeared after birth [15, 28]. As is well-known, although primordial germ cells of mouse embryos exhibit conspicuous alkaline phosphatase (ALP ase) activity [1], the reaction with ALPase in germ cells completely disappears after birth. The changes in ALPase activity during gonadal development are similar to those in binding patterns of some lectins (GS-I and BPA) [14]. Recently, EE2 antigen was found to localize on the cell surface of gonocytes from spermatogonia to preleptotene spermatocytes [19]. The antigenic molecule recognized by $\mathrm{mAb}$ EE2 is a novel glycoprotein with a molecular weight of $114 \mathrm{kDa}$. Due to its affinity with ConA and WGA lectins, and susceptibility to $\mathrm{N}$-glycanase, it is suggested that the glycoprotein contains asparagine-linked sugar chains. The functional significance of the epitopes recognized by these markers is unclear. However, it is interesting that the appearance of the determinant detected by them during germ cell development correlates with the period when gonocytes become arrested in their mitotic activity. In addition, the determinant disappears a few days before re-entry of the gonocytes onto mitosis. Hence, it is possible that the 


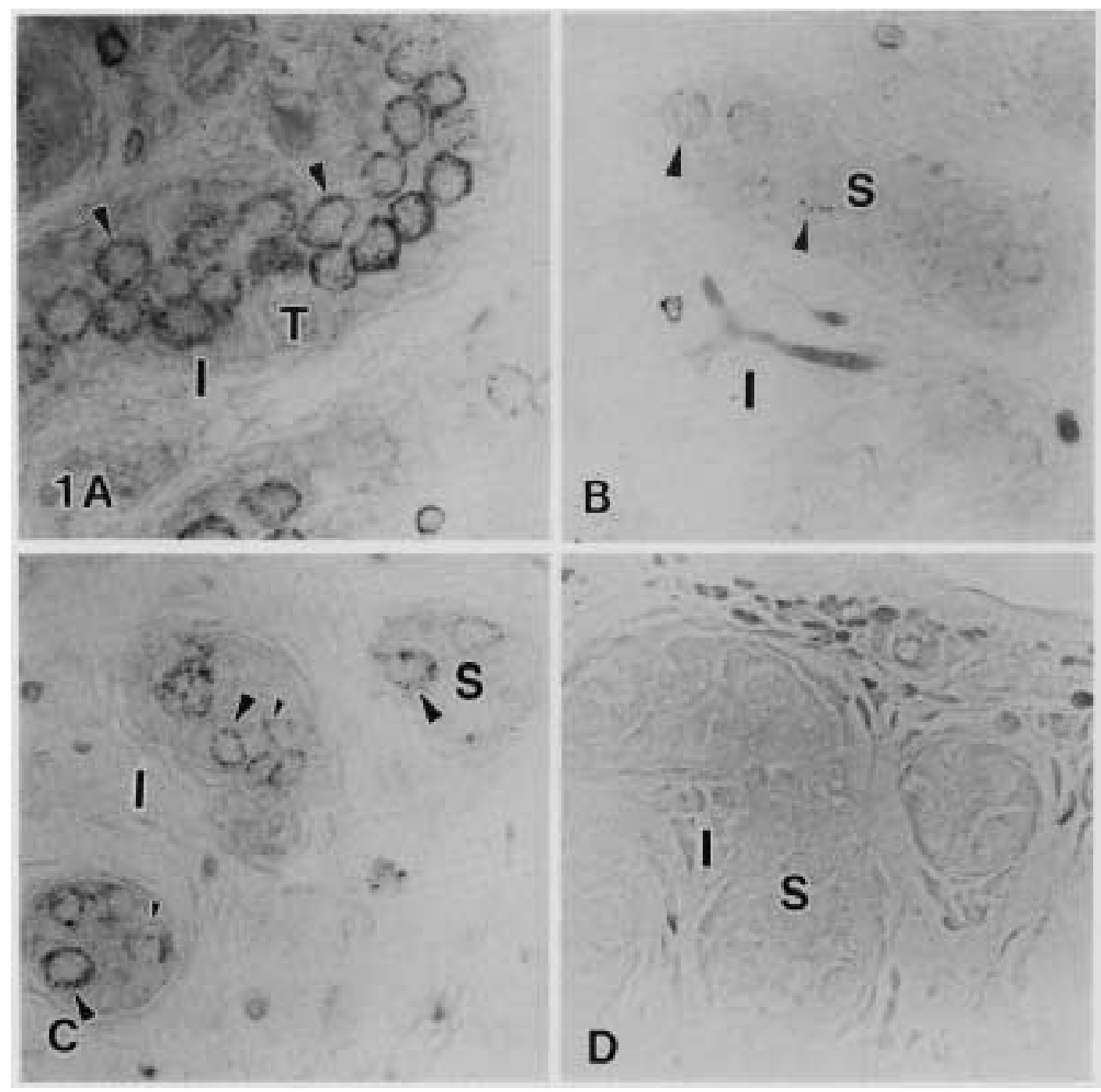

Fig. 1 A-D. Light micrographs showing sWGA bindings in the testis from 16.5 dpc to 4.5 dpp. Arrowheads: gonocytes, small arrowheads: Sertoli cells, I: interstitium, T: testicular cord, S:seminiferous tubule. A: 16.5 dpc. sWGA reacts in the cytoplasm, and plasma membrane of gonocytes B: 0.5 dpp. sWGA reaction decreases. C: 4.5 dpp. sWGA is detected in cytoplasm and plasma membrane of gonocyte. D: Control section incubated with biotinyl sWGA in the presence of $0.2 \mathrm{M} \mathrm{N}$-acetylglucosamine. Positive reaction is completely inhibited. $\times 480$ each.

appearance or disappearance of this determinant in gonocytes may be related to the differentiating process before the onset of spermatogenesis. Binding of FITClectins to gonocytes never changes after birth in the CD-1 strain mouse [2]. It is probable that lectin binding to germ cells may be different among strains [12]. In summary, sWGA, LEA and VVA are useful tools for studying the cell surface and cytoplasm carbohydrates of gonocytes during prespermatogenesis. The present findings indicate that there are changes in the glycosylation process of the VVA and sWGA-binding glycoconjugates during germ cell differentiation.
ACKNOWLEDGMENTS. The authors wish to thank Mr. Tsugiyama, I. (Department of Veterinary Anatomy, the University of Tokyo) for his expert care in keeping the laboratory animals. This work was supported in part by a grant-in-aid for Scientific Research Fund from the Ministry of Education, Science, Sports and Culture, Japan.

\section{REFERENCES}

1. Borghese, E. 1956. La fosfatasi alcalina nelle gonadi dol Mus musculus normale e dell' anemico W/W. Symp.Ggenetica. et Biologica. 5: 131-140.

2. De Felici, M. 1984. Binding of fluorescent lectins to the surface of germ cells from fetal and early postnatal mouse 


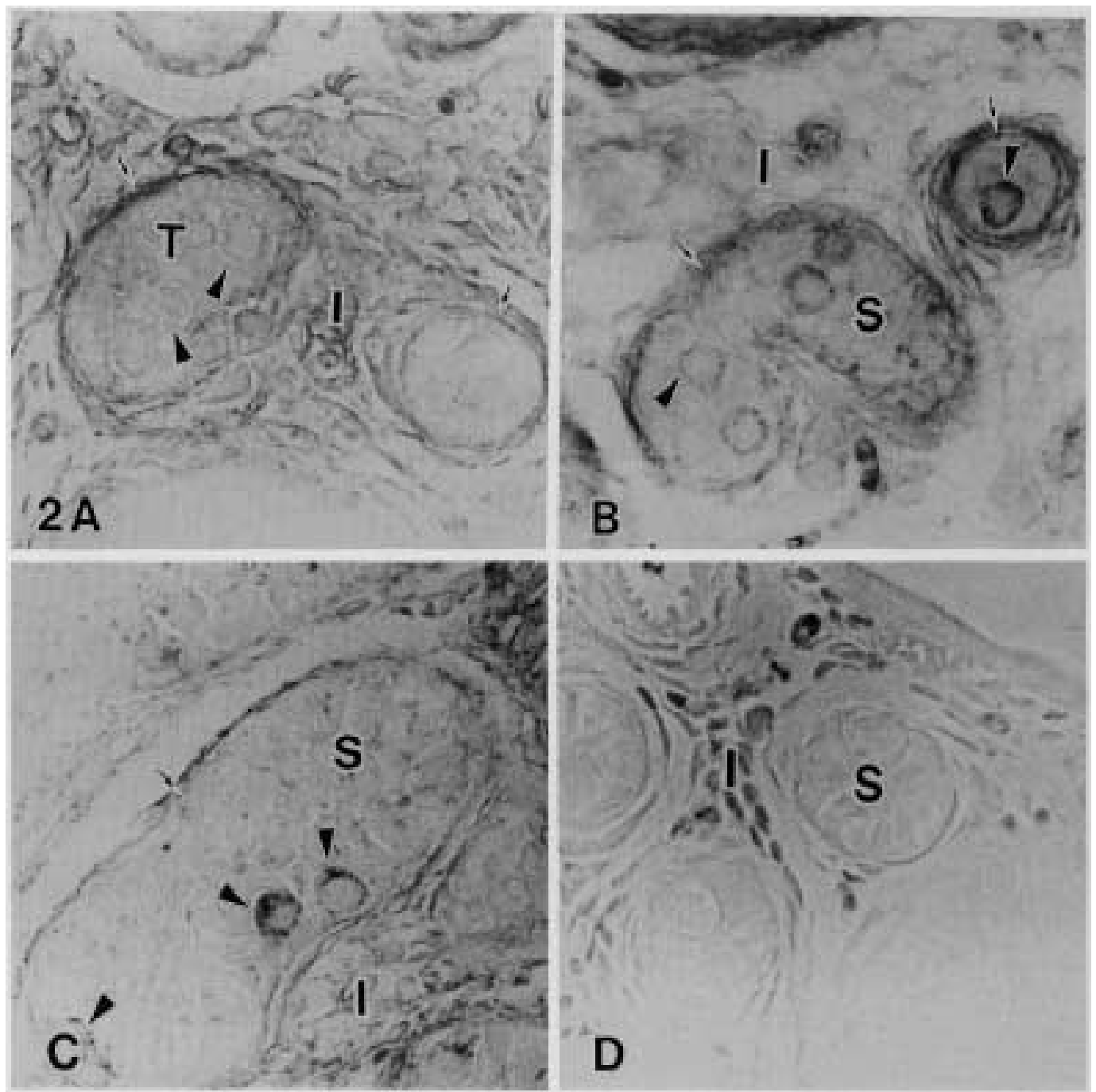

Fig. 2 A- D. Light micrographs showing VVA bindings in the testis from 16.5 dpc to 4.5 dpp. Arrowheads: gonocytes, small arrowheads: Sertoli cells, I: interstitium, T: testicular cord, S :seminiferous tubule. A: 16.5 dpc. VVA reacts only in a part of the interstitium. B: $0.5 \mathrm{dpp}$. VVA reaction is observed in the plasma membrane. C: $4.5 \mathrm{dpp}$. VVA reaction is observed in the plasma membrane and cytoplasm of gonocytes. D: Control section incubated with biotinyl VVA in the presence of $0.2 \mathrm{M} \mathrm{N}$-acetylgalactosamine. Positive reaction is completely inhibited. $\times 480$ each.

gonads. Gamete Res. 10: 423-432.

3. Fazel, A.R., Schulte, B.A. and Spicer, S.S. 1990. Glycoconjugate unique to migrating primordial germ cells differs with genera. Anat.Rec. 228: 177-184.

4. Fazel, A.R., Schulte, B.A., Thompson, R.P. and Spicer, S.S. 1987. Presence of a unique glycoconjugate on the surface of rat primordial germ cells during migration. Cell Differ. 21: 199-211.

5. Foster, J.D., Getchell, M.L. and Getchell, T.V. 1991. Identification of sugar residues in secretory glycoconjugates of olfactory mucosae using lectin histochemistry. Anat.Rec. 229: 525-544.

6. Fox, N., Damjanov, I., Hernandez-Martinez, A., Knowles, B.B. and Solter, A.D. 1981. Immunohistochemical localization of the early embryonic antigen (SSEA-1) in postimplantation mouse embryos and fetal and adult tissues. Dev. Biol. 83: 391-398.

7. Flickinger, C. 1967. The postnatal development of the Sertoli cells of the mouse. Z. Zellforsch. Mikrosk. Anat. 78: 92-113.

8. Franchi, L.L. and Mandle, A.M. 1964. The ultrastructure of germ cells in foetal and neonatal male rats. J. Embryol. Exp. Morph. 12: 289-308.

9. Gallagher, J.T. 1984. Carbohydrate-binding properties of lectins: a possible approach to lectin nomenclature and classification. Biosci. Rep. 4: 621-632.

10. Gondos, B. 1977. Testicular development. pp. 1-35. In :The Testis, vol. IV, Raven Press, New York.

11. Hahnel, A.C. and Eddy, E.M. 1986. Cell surface markers of mouse primordial germ cells defined by two monoclonal antibodies. Gamete Res. 15: 25-34. 


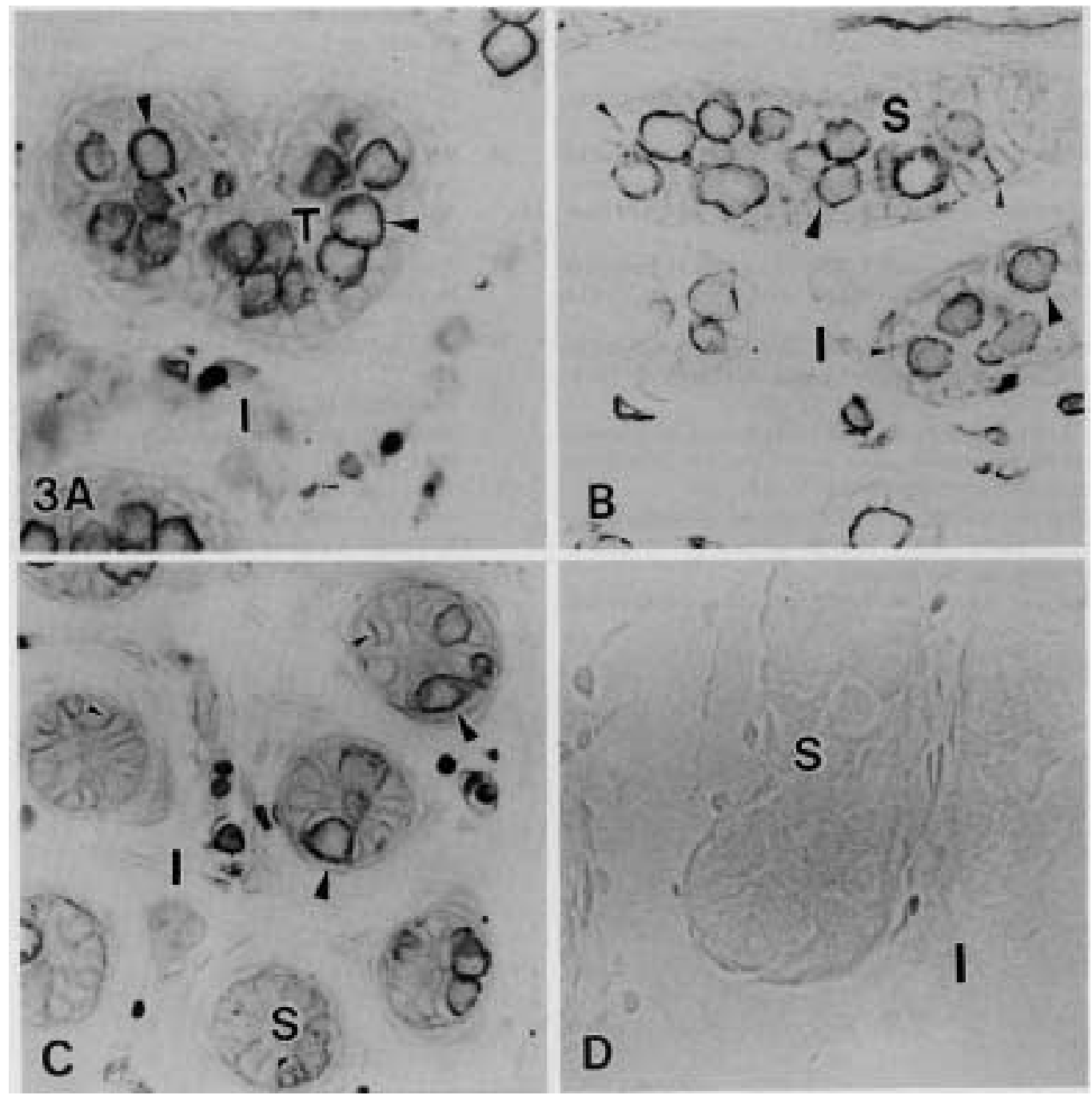

Fig. 3 A-D. Light micrographs showing LEA bindings in the testis from $16.5 \mathrm{dpc}$ to 6.5 dpp Arrowheads: gonocytes, small arrowheads: Sertoli cells, I: interstitium, T: testicular cord, S: seminiferous tubule. A: 16.5 dpc. LEA reacts strongly in the plasma membrane and cytoplasm of gonocytes. B: $0.5 \mathrm{dpp}$. LEA is weakly positive only in the plasma membrane of Sertoli cells and a part of the basement membrane in addition to gonocytes. C: $6.5 \mathrm{dpp}$. LEA reacts strongly in the plasma membrane and cytoplasm in gonocytes. D: Control section incubated with biotinyl LEA in the presence of $0.2 \mathrm{M} \mathrm{N}$-acetylglucosamine. Positive reaction is completely inhibited. $\times 480$ each.

12. Holm, M., Berger, A.E., Swanson, K. and Gonsberg, L.C. 1987. Effect of trypsinization on lectin binding to germ cells from ICR and T/t ${ }^{6}$ mice. Biol. Reprod. 37: 282-287.

13. Huckins, C. 1963. Changes in gonocytes at the time of initiation of spermatogenesis in the rat. Anat. Rec. 145: 243 (abstract).

14. Kanai, Y., Kawakami, H., Kurohmaru, M., Hayashi, Y., Nishida, T. and Hirano,H. 1989. Changes in lectin binding pattern of gonads of developing mice. Histochemistry 92: $37-42$.

15. Kanai, Y., Kawakami, H., Takata, K., Kurohmaru,M., Hayashi, Y., Nishida, T. and Hirano,H. 1990. Localization of Forssman glycolipid and GM1 ganglioside intracellularly and on the surface of germ cells during fetal testicular and ovarian de- velopment of mice. Histochemistry 94: 561-568.

16. Kanai, Y., Kawakami, H., Takata, K., Kanai-Azuma, M., Kurohmaru, M., Hirano,H., Hayashi, Y. and Nishida, T. 1992. Changes in intracellular and cell surface localization of Lex epitope during germ cell differentiation in fetal mice. J.Vet. Med. Sci. 54: 297-303.

17. Kluin, M., Kramer, M.F. and De Rooij, D.G. 1984. Proliferation of spermatogonia and Sertoli cells in maturing mice. Anat. Embryol. 169: 73-78.

18. Kluin, M. and De Rooij, D.G. 1981. A comparison between the morphology and cell kinetics of gonocytes and adult type undifferentiated spermatogonia in the mouse. Int. J. Androl. 4: 475-493.

19. Koshimizu, U., Nishikawa., H., Watanabe, D., Domae, K. 
and Nishimune, Y.1995. Characterization of a novel spermatogenic cell antigen specific for early stages of germ cells in mouse testis. Mol.Reprod. Dev. 40: 221-227.

20. Kretser, D.M. 1990. Germ cell-Sertoli cell interactions. Reprod. Fertil. Dev. 2: 225-235.

21. McGuinness, M. P. and Orth, J. M. 1992. Reinitiation of gonocyte mitosis and movement of gonocytes to the basement membrane in testes of newborn rats in vivo and in vitro. Anat. Rec. 233: 527-537.

22. Millette, C.F. and Bellve, A.R. 1977. Temporal expression of membrane antigens during mouse spermatogenesis. J. Cell Biol. 74: 86-97.

23. Muramatsu, T. 1988. Alteration of cell-surface carbohydrates during differentiation and development. Biochemie 70: 15871596.

24. Novi, A. M. and Saba, P. 1968. An electron microscopic study of the development of rat testis in the first 10 postnatal days. Z. Zellforsch. Mikrosk. Anat. 86: 313-326.

25. Orth, J.M. and Boehm, R. 1990. Functional coupling of neonatal rat Sertoli cells and gonocytes in coculture. Endocrinology 127: 2812-2819.

26. Sakamaki, K., Sawada, K., Koshimizu., U. and Nishimune,
Y. 1989. Identification of peanut agglutinin receptors on mouse testicular germ cells. Biol. Reprod.41: 1097-1102.

27. Sapsford, C.S. 1962. Changes in the cells of the sex cords and seminiferous tubules during the development of the testis of the rat and mouse. Aust. J. Zool. 10: 178-193.

28. Van Dissel-Emiliani, F.M.F., Van Kooten, P.J.S., De BoerBrouwer, M., De Rooij, D. G. and Van der Donk, J.A. 1993. A monoclonal antibody recognizing a differentiation marker on rat gonocytes. J. Reprod. Immun. 23: 93-108.

29. Vergouwen, R. P. F. A., Jacobs, S. G. P. M., Huiskamp, R., Davids, J. A. G. and De Rooij, D. G. 1991. Proliferative activity of gonocytes, Sertoli cells and interstitial cells during testicular development in mice. J. Reprod. Fertil. 93: 233243.

30. Wartenberg, H. 1976. Comparative cytomorphologic aspects of the male germ cells, especially of the "onia". Andrologia. 8: $117-130$

31. Yoshinaga, K., Muramatsu, H. and Muramatsu, T. 1991. Immunohistochemical localization of the carbohydrate antigen 4C9 in the mouse embryo: a reliable marker of mouse primordial germ cells. Differentiation 48: 75-82. 